\title{
Bone marrow necrosis in pediatric malignancies - 10-year retrospective review and review of literature
}

\author{
Michell Lozano-Chinga ${ }^{1}$, Lauren Draper ${ }^{2}$, Tracy George ${ }^{3}$, Archana Agarwal $^{4}$, David \\ Dansie $^{1}$, and Luke Maese ${ }^{5}$ \\ ${ }^{1}$ Primary Children's Hospital \\ ${ }^{2}$ Saint Louis University \\ ${ }^{3}$ The University of Utah School of Medicine \\ ${ }^{4}$ University of Utah \\ ${ }^{5}$ University of Utah School of Medicine
}

September 11, 2020

\begin{abstract}
Bone marrow necrosis (BMN) is a rare pathologic finding, but when encountered is most often associated with malignancy. In adults its presence correlates with an inferior prognosis, however in children the prognostic implication is unclear. We performed a retrospective review of 3,760 bone marrow specimens in patients [?] 18 years over a ten-year period. BMN was identified in less than $1 \%$ of specimens and only in patients with leukemia, lymphoma or neuroblastoma. BMN contributed to a delay in diagnosis; however, advanced medical imaging may serve as a tool to localize non-necrotic areas for bone marrow sampling, facilitating an expedited diagnosis.
\end{abstract}

\section{Introduction}

Bone marrow necrosis (BMN), defined as the death of the medullary stroma with preservation of the cortical bone, is a rarely reported finding in the pediatric population. There are various etiologies for BMN including medications, radiation, infection, autoimmunity, and sickle cell disease. However, the vast majority ([?]99\%) are associated with malignancies. BMN is encountered more frequently in adults, documented in up to $5.6 \%$ of initial diagnostic acute leukemia bone marrow specimens, and is associated with an inferior prognosis. BMN in the pediatric population contributes to a challenging diagnostic process, and its effect on cancer prognosis remains unknown. A prior review described five cases of acute leukemia and two cases of neuroblastoma related to BMN over a 10-year period. A separate review noted 12 patients with acute lymphoblastic leukemia (ALL) and concomitant BMN as part of their initial presentation. In a 10-year retrospective review performed at the University of Utah, we identified nine bone marrow biopsies from six different pediatric patients with BMN, all associated with malignancies.

\section{Methods}

The study was approved by the institutional review and ethics boards at the University of Utah. We queried the electronic database from the Associated Regional and University Pathologists (ARUP) Laboratories for all cases of "bone marrow necrosis", "acute lymphoblastic leukemia", "acute myeloid leukemia" and "neuroblastoma" in patients that were 18 years and younger from January $1^{\text {st }}, 2010$ to February $1^{\text {st }}, 2020$. ARUP Laboratory is an enterprise of the University of Utah and its Department of Pathology. It serves as a not-for-profit national reference laboratory. 
The clinical, laboratory and radiological information of the patients were obtained from electronic medical records.

\section{Results}

During a 10-year period, 3,760 bone marrow samples were evaluated. The samples were made up of ALL, acute myeloid leukemia (AML), and neuroblastoma diagnoses at $35 \%(\mathrm{n}=1,298), 9 \%(\mathrm{n}=344)$, and $2 \%$ $(\mathrm{n}=77)$, respectively.

During this time, nine samples $(0.24 \%)$ in six unique patients, all with malignancy, were identified to have BMN (Table 1). The mean age was seven years (11 months - 18 years). Four patients were female and two were male. One patient had relapsed/refractory B-ALL, one had AML, three had neuroblastoma, and one had diffuse large B-cell lymphoma (DLBCL). Eight bone marrow evaluations were performed prior to chemotherapy and one was performed after reinduction therapy.

Hematologic laboratory findings were obtained from eighth encounters. The mean hematologic laboratory findings were leukocytes of $6.9 \times 10^{9} / \mathrm{L}$ (range $0.3-13.3 \times 10^{9} / \mathrm{L}$ ), hemoglobin of $9.7 \mathrm{~g} / \mathrm{dL}$ (range 8.3-11.5 $\mathrm{g} / \mathrm{dL})$, and platelets of $336 \times 10^{9} / \mathrm{L}\left(38-667 \times 10^{9} / \mathrm{L}\right)$. Severe neutropenia and thrombocytopenia were evidenced only in the patient with refractory B-ALL. The patient with AML initially had normal peripheral blood counts when the first bone marrow sample was obtained, however with subsequent assessments there was gradual development of leukopenia and neutropenia. Neither of the patients with leukemia had peripheral blasts at the time of bone marrow evaluation. All patients with neuroblastoma had normal blood counts at the time of bone marrow assessment and the majority had extensive tumor involvement in the marrow ranging from $60 \%$ to $>95 \%$. Three of our patients required repeat bone marrow evaluations due to either an inability to establish a diagnosis, or a suboptimal initial sample.

In regard to systemic symptoms, all patients had musculoskeletal symptoms manifested as lower extremity and/or back pain. This was the most common initial complaint and prompted an expanded workup, with five of six patients undergoing imaging. Plain radiographs were unremarkable; however, other imaging modalities including bone scan, magnetic resonance imaging (MRI), and computed tomography were abnormal demonstrating increased uptake in the bone marrow of long bones, lytic lesions and/or sclerotic bone.

Follow up was documented in the electronic records for all patients treated in our institution. The patient with refractory B-ALL died from vancomycin-resistant enterococcus sepsis. Four patients are in remission after chemotherapy treatment (2-5 years) (Table 1$)$. Repeat bone marrow examinations showed no evidence of necrosis on follow up assessment for both the AML (end of induction) and neuroblastoma (4-7 months of treatment) patients.

\section{Discussion}

BMN is an exceedingly rare pathologic finding in children. In our review of 3,760 bone marrow studies, six patients $(0.24 \%)$ were identified to have BMN, all in the setting of malignancy. Based on the rarity of this pathologic finding, and the strong association with malignancy, a high suspicion should be maintained in pediatric patients with BMN.

Several themes emerged in the presentation, evaluation, and outcomes in the review of our cohort. The common presenting symptom for all patients was pain which prompted further evaluation. A review of peripheral blood findings, similar to a previous study, showed anemia as the predominant cytopenia, with leukopenia and thrombocytopenia developing later.

In the evaluation of BMN, diagnostic radiological imaging can serve as a helpful resource. MRI has been widely used to detect bone abnormalities in multiple disease entities, including hematologic malignancies. Previously described BMN MRI findings include fluid-filled necrotic lesions, hyperintense lesions and other findings with similar appearance to periarticular avascular necrosis. While not typically part of the diagnostic workup, due to atypical presentations of patients in our cohort, MRIs were obtained in many patients demonstrating abnormal bone signal corresponding to areas of BMN. Patient 2, after undergoing two previous 
non-diagnostic bone marrow examinations at the bilateral ilia, underwent an MRI which demonstrated absence of necrosis at the humerus and tibia. This prompted a BM evaluation at these sites which yielded viable tissue and ultimately lead to the diagnosis of AML (Figure 1). In patients with BMN evidenced by biopsy, imaging provides the advantage of analyzing a larger area of bone and subsequently determining the best place to biopsy and obtain viable tissue.

In regards to outcomes, only one patient in our cohort (Patient 1) died. Patient 1 was an outlier as BMN was not identified on initial diagnostic bone marrow evaluation and only identified after receiving intensive multi-modal chemotherapy. The remainder of evaluable patients are in disease-free remissions of two years or more. Based on ours and other previous studies, inferior outcomes in the setting of BMN have not been realized in the pediatric population, making it an unlikely indicator of prognosis. This is in contrast to adults where BMN necrosis has been associated with poor outcomes

An additional finding upon review of patients that required repeat bone marrow evaluations revealed they had previously been treated with immunosuppressive therapy, including steroids and a tumor-necrosis-factor antagonist. Based on the small sample, it is unclear if this played a role in the development of BMN. Further studies are needed to assess a potential causal relationship between immunosuppressive medication in the setting of malignancy and BMN.

While this review encompassed more than 3,700 bone marrow evaluations, it is a single institutional review which limits the scope. Additionally, comprehensive chart review from one case was not completed due to an inability to access outside medical records.

BMN in the pediatric population is exceedingly rare and when encountered, a malignant etiology should be suspected. Diagnosis from bone marrow evaluations is challenging, and radiological imaging is a helpful modality for localizing non-necrotic areas of bone marrow. A more comprehensive multi-institutional review would allow for a greater understanding of this unique pathologic entity.

\section{References :}

FIGURE 1 Bone marrow aspirate (A) showing myeloblasts. Bone marrow biopsy (B) consistent with bone marrow necrosis in the patient with AML. Axial T2 (C) and T1 (D) post-contrast images demonstrate a lesion in the left iliac bone consistent with bone marrow necrosis (white arrow). 


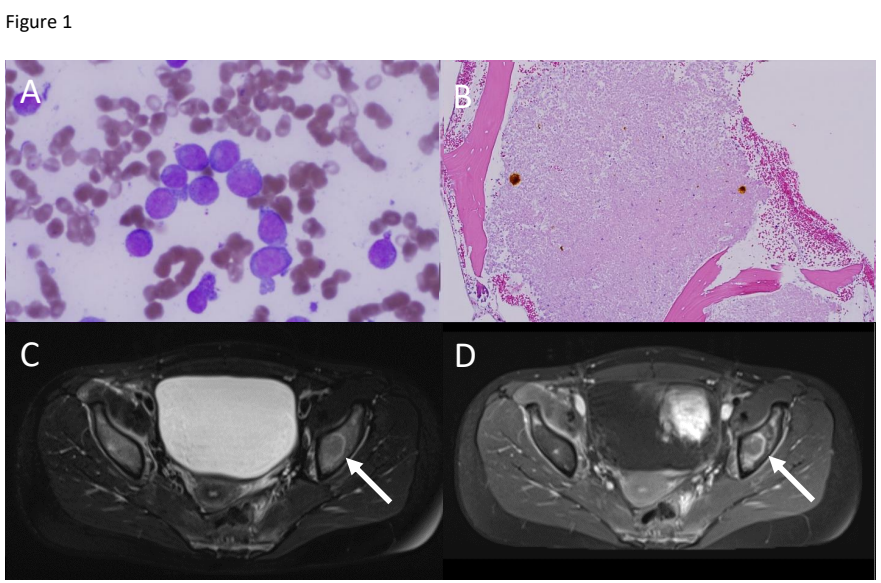

\section{Hosted file}

Table 1. Clinical laboratory and radiological characteristics.docx available at https: //authorea.com/users/357891/articles/480267-bone-marrow-necrosis-in-pediatricmalignancies-10-year-retrospective-review-and-review-of-literature 